Jens Sambale, Dominik Veith

\title{
Berliner Wagenburgen: Transformation peripherer Räume, Stigmatisierung sozialer Gruppen und die Abwehr von Marginalisierung
}

»Der städtische Raum ist konkreter Widerspruch»(Roger Keil, 1993: 305)

Wagenburgen, Spontansiedlungen, mobile Wohnformen, Zeltstädte, Obdachlosigkeit in allen Schattierungen und viele andere Unterbringungsformen jenseits des Normalmietverhältnisses sind urbane Wachstumsphänomene und keine der Schrumpfung. Begriffe wie De-Urbanisierung, Exurbanisierung oder Suburbansierung kennzeichnen eine konkurrierende Realität der Stadtentwicklung (Garreau, 1991, Soja 1992, Ronneberger 1997b). Die Gleichzeitigkeit der Bevölkerungszunahme in den urbanen Zentren und der Ausdehnung der bebauten Fläche an den städtischen Rändern, scheint das klassische Urbanisierungsmodell der Einheit von sozialer und räumlicher Mobilität zu bestätigen: Danach erfolgt die Integration von ZuwanderInnen in die Stadt über die Arbeits- und Wohnungsmärkte in den Zentren und findet ihren Abschluß idealerweise in der Eigentumsbildung am Stadtrand. Arbeitsverhältnisse mit tariflichem Lohn und garantierten sozialen Leistungen sowie Mietverhältnisse mit gesetzlichen Standards und Ansprüchen münden in der bürgerlichen Kernfamilie mit Wohneigentumswunsch. $\mathrm{Da} 3$ dieses wachtstumsorientierte Modell sozialer und räumlicher Mobilität immer schon auf dem selektiven Ein- und Ausschluß Anspruchsberechtigter (Becker 1997) gegründet war und daß die gegenwärtige Krise der Arbeits- und Wohnungsmärkte von Ausgrenzungsprozessen gekennzeichnet ist, können wir hier nicht weiter thematisieren. Im Unterschied zu Obdachlosenunterkünften und anderen prekären Unterbringungsformen, die das Scheitern der Wohnraumversorgungspolitik kennzeichnen, formulieren Wagenplätze eine aktive Kritik an der Warenförmigkeit städtischen Lebens und den diziplinierenden Zumutungen der Normalbiographie. Mit der Transformation (West-) Berlins von der urbanen Ausnahme zur metropolitanen Kapitale wandelt sich die Funktion der Wagenburgen für die städtische Reproduktion: An die Stelle des Zeugnisses Westberliner Toleranz 
und Offenheit gegenüber kollektiven Lebensformen tritt die städtische Feinderklärung. Gegenstand des Textes ist dieser Funktionswandel, der eng an die verwertungsorientierte Aufwertung ehemals peripherer Räume gebunden ist sowie die taktischen und strategischen Maßnahmen der WagenbewohnerInnen gegen die Politik der Stigmatisierung.

\section{Restrukturierung der sozialräumlichen Ordnung}

"Bitte bleiben Sie in Berlin. Bleiben Sie Berliner (Bausenator Klemann auf den Schautafeln zu der Ausstellung Eigentum 2000, 1997)

Die oben angedeutete Gleichzeitigkeit von Bevölkerungszunahme im Zentrum und der Ausdehnung der Wohngebiete an der städtischen Peripherie beiderseits der Stadtgrenze, scheint für Berlin nicht zuzutreffen. Stagnation und Krise bestimmen die Rhetorik städtischer Eliten. Die Berliner Besonderheit der Einheit von Siedlungsgebiet und Gebietskörperschaft ${ }^{1}$ weicht dank steuerlicher Vergünstigungen einem uniformen Siedlungsbrei von Wohn- und Gewerbeparks jenseits der Stadtgrenze, auf die Berliner Planungsbehörden kaum Zugriff haben. Diese unkontrollierte Ausdehnung urbaner Randgebiete artikuliert einen Prozeß sozialen Wandels, der mit dem Begriff nachholender Suburbanisierung nur unzureichend beschrieben ist. Trotzdem kann in Berlin keineswegs von einer Verödung des geographischen Zentrums gesprochen werden. Die Arbeitsplatz- und Bevölkerungsdichte liegen mit 1,2 Millionen EinwohnerInnen und 800.000 Arbeitsplätzen in der Innenstadt deutlich höher als im Rest der Republik (Senatsverwaltung für Wirtschaft und Betriebe 1995: 9). Auch EinwanderInnen- und Arbeitslosenquartiere wie der Bezirk Kreuzberg wachsen allen Abwanderungsprognosen zum Trotz bei sinkendem Wohnungsbestand ungebrochen weiter. Allerdings siedelt hier immer weniger der ökonomisch potente Stadtbürger (vgl. Heeg im vorliegenden Band) als vielmehr eine wachsende Zahl diskriminierter Bevölkerungsgruppen.

Die Suburbanisierung von Produktion, Handel und einkommensstarken Haushalten über die Stadtgrenze hinweg, führt zur Reorientierung lokaler Politik. Die Senatsverwaltung für Stadtentwicklung, die Bauverwaltung und die Wohnungswirtschaft reagieren mit konkurrierenden Maßnahmen auf diesen Verlust von WählerInnen, MieterInnen und SteuerzahlerInnen. Zur Sicherung der »strukturellen Stabilität« der Stadt, so Staatssekretär Ba-

1 Im Westteil verhinderte die Mauer die Ausdehnung des Siedlungsgebietes über die Stadtgrenze hinaus. Auch im Osten fand extensiver Wohnungsbau fast ausschließlich innerhalb der Stadtgrenze an der östlichen Peripherie statt. Der Anteil des privaten Wohnungsbaus in und um Berlin fällt weit hinter den nationalen Durchschnitt der DDR und dem anderer RWG-Staaten zurück (vgl. Schulz 1991). Beiden Stadthälften war die Ausdehnung der Gebietskörperschaft völkerrechtlich untersagt. 
roner (1996: 2), legt die Bauverwaltung eine Eigentumsoffensive ${ }^{2}$ am Stadtrand auf, der Stadtentwicklungssenator publiziert das Planwerk Innenstadt ${ }^{3}$, das die City als Standort für den immmobilienbesitzenden Stadtbürger erschließen soll und die Wohnungswirtschaft setzt eine Reihe von Maßnahmen zum Schutz ihrer Investitionen durch. So sieht u.a. das Maßnahmenprogramm zur Sicherung und Verbesserung des Sozialgefüges im Sozialwohnungsbestand der Großsiedlungen in elf Gebieten ab April 1998 vor: Auf die Erhebung der Fehlbelegungsabgabe zu verzichten, die Belegungsbindungen aufzuheben und Besetzungsrechte nicht mehr wahrzunehmen. Damit entgeht dem strapazierten Budget Berlins nicht nur eine Einnahmequelle, der lokale Staat verzichtet darüber hinaus auf eine der wenigen Möglichkeiten, diskriminierte Gruppen mit Wohnraum zu versorgen.

Die politische Orientierung an den Mittelklassen und ihre polizeiliche wie private Absicherung markieren Versuche, die Zentralität Berlins als Hauptstadt und Standort zu stärken. Immer wieder betont das Planwerk das Primat der Mitte (taz 28.11.96). Bevölkerungsgruppen und Nutzungen, die sich nicht in dieses neue städtische Leitbild fügen können (Obdachlose und Arme) oder wollen (Wagenplätze, HausbesetzerInnen) werden marginalisiert und an die städtische und soziale Peripherie gedrängt. Polizei, Sicherheitsdienste und die verschiedenen Senatsverwaltungen agieren als Raumpatrouille, die akzeptierte Nutzungen und Verhaltensweisen in städtischen Teilräumen definiert und sanktioniert. Dieser Privilegierung der räumlichen Form über soziale Prozesse liegt die Vorstellung zugrunde, daß innerhalb einer fixierten räumlichen Gestalt durch geschicktes Management der planenden Verwaltung soziale Prozesse gesteuert und kontrolliert werden können. Diese räumliche Kontrollfunktion leitet nicht allein das Planwerk Innenstadt und die Eigentumsoffensive, sondern auch die Vertreibungsprozesse in den Innenstädten und die NIMBY-Revolten ${ }^{5}$ an der urbanen Peri-

2 Der Angriffscharakter gegen MieterInnen kommt in der Maßnahme zur Geltung: Es ist nicht die »Emanzipation der Mieter « (Titel Stadtforum, Mai 1997), sondern die »Emanzipation von dem Mieter« (Titel MieterEcho 263/97), die hinter der Offensive steht (zur Mittelklasseorientierung dieser Maßnahmen siehe Heeg in diesem Band, für eine generelle Kritik der Eigentumsbildung in Berlin siehe Wolff 1996).

3 Zum Planwerk vgl. Senatsverwaltung für Stadtentwicklung 1997a, kritisch u.a.: Architektenkammer (1997), Hain (1996), Tageszeitung/Scheinschlag (1996), Schmals/Jahn (1997).

4 Die Maßnahmen sind auf zunächst drei Jahre befristet. Das Maßnahmenprogramm steht unmittelbar vor der Verabschiebung (Korrespondenz mit der Senatsverwaltung für Bauen, Wohnen und Verkehr, Januar 1998, vgl. AH 1996f).

$5 \quad N I M B Y$ ist das angelsächsische Akronym für: Not In My Back Yard. NIMBY-Strategien haben sich in den USA als ausgesprochen erfolgreich bei der Abwehr unerwünschter Nutzungen in Eigenheimsiedlungen erwiesen. Die Definition unerwünschter Nutzungen wird dabei immer weiter ausgedehnt. Handelte es sich klassisch um Einrichtungen der technischen (Müllverbrennungsanlagen) und der sozialen Infrastruktur (Obdachlosenhilfe, forensische Kliniken) werden mittlerweile selbst Apotheken als potentielle Anbieter von Spritzen für DrogenkonsumentInnen an der Ansiedlung gehindert. Ratio dieser Politik ist 
pherie (s.u.). Die Errichtung polizeilicher Verbotszonen und Zugangsbarrieren gegenüber den unwürdigen Armen, die kontinuierliche Ausweitung des Hausrechtes über ehemals öffentliche Räume (Innen!Stadt!Aktionen! 1997), ${ }^{6}$ die Abschottung wohlhabender Haushalte von den Konsequenzen polarisierter Einkommens- und Vermögensverteilung ${ }^{7}$ usw. versuchen, die urbane Geographie der Ungleichheit zu organisieren. Diese Geographie ist nicht eindeutig fixiert. In diesem Prozeß ist Marginalität keine statische Kategorie der Sozialstruktur, sondern eine dynamische Strategie lokaler Eliten (Sambale/Veith 1997). Die räumliche Konzentration stigmatisierter Gruppen, nicht ihre konkrete (Über-)Lebenssituation, nährt Ghettophantasien und schürt eine erregte Angstlust beim Publikum.

In diesen komplexen und widersprüchlichen Prozessen der Territorialisie-

der Erhalt und die Steigerung des Bodenwertes, Resultat ist die ethnische Risikodiskriminierung in den Siedlungsgebieten der Armen und EinwanderInnen, denen diese Nutzungen aufgebürdet werden (Sambale 1994). Auch wenn es NIMBYism von MieterInnen zur Verteidigung der Lebensqualität gibt, halten wir den Klassencharakter und die Verräumlichung ungleicher sozialer Beziehungen für das bestimmende Kriterium. Von daher scheidet der Begriff des Sankt-Florian-Prinzips aus.

6 Der Berliner Los-Angeles-Platz, im Windschatten des Kurfürstendammes artikuliert die weitreichenden sozialen Konsequenzen, die ein geringfügiger Wechsel im Eigentumsregime zeitigt. Unter dem als öffentliche Grünfläche ausgewiesenem Platz betrieb die AG City, der Zusammenschluß des Handelskapitals entlang des Kurfürstendammes, im Auftrag des Berliner Senates ein Parkhaus gegen geringes Entgelt, um automobile KundInnen in der Innenstadt zu halten. Die Intensivierung der Kontrollen am Kurfürstendamm trieb marginalisierte NutzerInnen in den Park. Mit der Veräußerung des Parkhauses an einen verwertungsorientierten Investor, gegen den Einspruch der AG City (Berliner Zeitung, 21.01.1997), wurde die Grünfläche privatisiert. Der neue Eigner schöpfte die Möglichkeiten des Hausrechtes umgehend aus. Alltagspraxen wie Essen, Trinken und Ballspielen sind untersagt. »Wir wollen Penner und Drogenabhängige vertreiben« verlautbart der Geschäftsführer (Berliner Morgenpost, 26.03.1997). Die dankbare Methaphorik des privatisierten Los Angeles Platzes auf dem Dach einer Tiefgarage mobilisierte zwar den Protest städtischer Gruppen, die im Rahmen der Innen!Stadt!Aktion! 1997 ein öffentliches Picknick auf dem Platz veranstalteten, geändert hat sich an den Zutrittsbarrieren indes nichts (Grell/Sambale/Veith 1997).

7 Das Unternehmen Groth\&Graffs plant am zentralen Klingelhöferdreieck eine Luxuswohnanlage mit Sicherheitszonen und Wachdiensten für Manager, Geschäftsleute und Botschaftsangehörige. Eine geplante öffentliche Kita, die die Kinder des einkommensschwachen Bezirkes Tiergarten auf das Gelände bringen würde, lehnt der Bauherr ausdrücklich ab (Tagesspiegel 22.6.1996).

8 Legende wurde Ende 1997 ein Artikel im Spiegel über Neukölln (Endstation Neukölln), der pauschal die Arbeitslosen und EinwanderInnen des größten Berliner Bezirkes als Frauenschläger und Kriminelle diskriminierte. Die diskursiven Auf- und Abwertungen städtischer Teilräume bereiten deren materielle Auf- und Abwertung vor und haben eine lange Tradition in Westberlin (Bodenschatz 1987). Sie sind Bedingung und Folge der Kapitalbewegungen im städtischen Raum. Anfang der 90er Jahre setzte nach langen Jahren der Sanierung ein publizistisches Kreuzberg-dreschen (z.B. taz 10.1.97) ein, das sich mit der Konsolidierung der Wohnungsmärkte im Prenzlauer Berg und in Mitte intensivierte. Gegenwärtig deutet sich eine zögerliche Renaissance des Bezirkes für die HIPeoisie (Smith 1996a: 33) an. So werden verlassene Fabriketagen kapitalintensiv in Lofts mit eigener Servicestruktur für »gehobene Ansprüche« entwickelt (taz 10.1.98, Tagesspiegel 28.12.97). 
rung sozialer Beziehungen in Berlin übernahmen die Wagenplätze und besetzten Häuser unfreiwillig die Rolle der städtischen Feinde.

\section{Wagendörfer und der Bedeutungswandel urbaner Räume}

»Die Wagenburgen sind im Schatten der Mauer entstanden. Jetzt werden die kostbaren Innenstadtbereiche für eine andere Nutzung benötigt« (Norbert Schmidt, Senat für Inneres, taz, 31.07.1995)

Wagenburgen oder der weniger defensive Terminus Wagenplätze, sind kollektive Wohnformen in unterschiedlichen, zumeist stillgelegten Anhängern oder Fahrzeugen (Zirkus- und Bauwagen, Wohnwagen, Lastwagen, teilweise PKW). ${ }^{9}$ Wir werden im folgenden die defensiven und sozial deklassierten Zufluchtsräume der WagenbewohnerInnen Wagenburgen nennen und die sozial stabilen, politisch aktiven Siedlungen Wagenplätze oder Wagendörfer. ${ }^{10}$ Die Unterscheidung ist von uns und reflektiert weder das Selbstverständnis der BewohnerInnen noch die Wahrnehmung der Medien oder der Verwaltung. Obwohl die Mehrzahl der Wohneinheiten mit Rädern versehen ist, sollten die Wagendörfer nicht mit mobilen Lebensformen wie jenen der Sinti und Roma oder der Wander- und SaisonarbeiterInnen verwechselt werden. ${ }^{11}$ Auch der Vergleich mit den mobile homes Nordamerikas trifft nicht zu. Zwar ähnelt sich die stadträumliche Form, doch die normativen Vorstellungen der BewohnerInnen und ihr Verhältnis zur Mehr-

9 Analytische Literatur zum Thema Wagenplätze ist rar. Eine Studie wurde im Rahmen des Nationalberichtes zur HABITAT II Konferenz erstellt, die wegen der Räumungen überholt ist, doch immer noch zitiert wird (Knorr-Siedow/Willmer 1994, Bundesministerium für Raumordnung, Bauwesen und Städtebau 1996). In der Bauwelt (Schubert 1997) und in der $A K$ (5.5.1993) sind weitere Texte erschienen. Ein Mitglied des Berliner Wagenburgenplenums hat einen längeren Beitrag geliefert (Berg 1997). Es liegen einige unveröffentlichte Diplomarbeiten vor. Die Arbeit von Kropp (1997) mit einer ausführlichen Bibliographie ist über das Wäglerarchiv verfügbar (http://www.bis.uni-oldenburg.de/ kropp). Weiter stützen wir uns auf verschiedene Selbstdarstellungen und Flugblätter der Wagenplätze (Wagenburg am Kinderbauernhof 1996, Wagenburg am Schwarzen Kanal 1997, Wagendorf Lohmühle 1997, Plenum der Berliner innerstädtischen Wagenplätze 1997a, b, c) sowie Gespräche mit den BewohnerInnen. Das Abgeordnetenhaus von Berlin liefert umfangreiche Dokumente (AH 1996-1997). Presseerklärungen der Senatsinnenverwaltung (1996a, b), der CDU (1996) und der Oberfinanzdirektion (1996) schließen die meisten Lücken. Alle Berliner Zeitungen haben ausführlich über den Konflikt berichtet. Die BI West-Staaken (1996) überließ uns eine Informationsbroschüre und stand für Nachfragen ebenso zur Verfügung wie die Bezirksverordnetenversammlung Spandau (1996).

10 Diese Trennung ist analytisch, sie dient nicht dazu, die bösen Wagenburgen von den guten Wagenplätzen zu scheiden. Der Wandel eines Platzes oder Dorfes zur Burg wird durch externe Einflüsse erzwungen. Der Unterschied erscheint uns nur sinnvoll zur Beschreibung von Differenzierungen innerhalb der baulichen Form Wagensiedlung. Wir beschränken uns auf die Berliner Wagenplätze, die in den 80er Jahren im Westteil der Stadt entstanden sind.

11 Die Haltung, die diesen Gruppen entgegenschlägt, ist ähnlich ablehnend. So ist in Berlin ein dauerhafter Platz für ortsfremde Sinti und Roma immer wieder umstritten. Das gleiche gilt für irische und britische WanderarbeiterInnen (taz 22.7.96). 
heitsgesellschaft unterscheiden sich erheblich. ${ }^{12}$ In Nordamerika handelt sich nicht um alternative Lebensentwürfe, sondern um Anpassungsstrategien an den Arbeitsmarkt.

Einzelne WagenbewohnerInnen mögen von Zeit zu Zeit reisen oder den Standort wechseln, doch die Kollektive hängen von der Dauerhaftigkeit der Plätze ab, um den Kontakt zum lokalen Arbeitsmarkt, zum Schul- und Ausbildungssystem aufrechtzuerhalten, um Verbindungen in die umliegende Nachbarschaft zu reproduzieren und die Notwendigkeiten und Annehmlichkeiten des täglichen Lebens jenseits der garantierten städtischen Verund Entsorgungsinfrastruktur zu organisieren.

Wagendörfer umfassen in der Regel nicht mehr als 25 EinwohnerInnen mit heterogenem Einkommens- und (formalem) Bildungsniveau - von vollzeitarbeitenden ArchitektInnen bis zu SozialhilfeempfängerInnen. Eine Studie von 1994 kommt zu dem Schluß, daß die Mehrheit der BewohnerInnen sich hinsichtlich sozialer Kompetenz und Einkommensquellen nicht von der Wohnbevölkerung vernachlässigter Gebiete unterscheide; je $20 \%$ werden den dauerhaft Wohnungslosen und der jugendlichen Punkszene zugerechnet (Knoor-Siedow/Willmers: 22-34). Mehr als jede dritte BewohnerIn bezog ihr Einkommen 1994 aus einem Arbeitsverhältnis oder Arbeitslosengeld, ebenfalls mehr als ein Drittel lebt von Sozialhilfe und insbesondere die Wohnungslosen und Punks müssen betteln - »in der Regel auch eine zeitaufwendige Tätigkeit« (ebd., 31). Das Problem dieser Kategorisierungen liegt in der erheblichen Differenzierung der einzelnen Plätze, die solche Generalisierungen nicht zulassen. Die Räumungen der sozial schwächsten Plätze nach 1994 hat die durchschnittliche Sozialstruktur der Plätze nachhaltig verändert. Die innerstädtischen Plätze liegen zum Teil deutlich über den soziökonomischen Werten ihrer behausten Nachbarn. Die WagenbewohnerInnen selbst schreiben: »Die einzelnen Plätze in Berlin sind recht unterschiedlich. ... Die größte Gruppe unter den WagenbewohnerInnen sind wohl JobberInnen und Leute, die sich in einer Ausbildung befinden « (Plenum 1997c: 4). Damit leugnen sie nicht die Präsenz hilfsbedürftiger WagenbewohnerInnen, sondern betonen, daß diese Menschen unterschiedlich auf die Plätze verteilt sind. Entscheidend ist, daß die jeweilige Gruppe sozial stabil ist: »Wenn es jedoch eine funktionierende Gruppe ist, dann wird der Fürsorgebedarf - sofern vorhanden - von dieser abgedeckt « (ebd.). Wagenplätze sind keine Wohnform Jugendlicher, die Mehrheit der BewohnerInnen ist mit deutlichen Streuungen 25 - 35 Jahre alt.

Der Anschluß an die städtische Infrastruktur ist durch unterschiedliche Mittel

12 Auch die vehiculary housed communities (Stefan 1997) und die selbstverwalteten Obdachlosensiedlungen der USA (Dear/Mahs 1994, 1997) sind nicht aus der Entscheidung für eine alternative Lebensform entstanden, sondern aus der Not geboren. 
gewährleistet, die idealerweise dem Anspruch der BewohnerInnen auf eine Verzichtsökologie nach den Prinzipien des sparsamen Gebrauches gerecht werden: »Es gibt Solarzellen, Regenwassernutzungen, Schilfkläranlagen, Kompostklos, Bepflanzungen...« (ebd.). Wasser muß in Kanistern transportiert werden und kommt wie die Stromversorgung häufig aus der Nachbarschaft - mal gegen Geld, mal nicht. Mit der Verlagerung der Plätze fallen solche Vereinbarungen weg. Die Post kommt oft direkt an den Wagen, Telekommunikation wird mit dem Handy geführt. Es gibt BewohnerInnen mit e-mail-Adressen. Die Rede- und Diskussionsbeiträge der Berliner Wagentage (s.u.) 1996 wurden im Internet publiziert. Handygebrauch und Internetkompetenz sind einige der Attribute, die auch den Stadtbürger zieren.

Entscheidungen, z.B. über neue MitbewohnerInnen, werden in der Regel auf dem Plenum des jeweiligen Platzes konsensual getroffen. Aus diesem Entscheidungsprinzip ergibt sich eine überschaubare Gruppengröße von maximal 25 Personen und notwendigerweise Selbstverwaltung, da ein übergeordneter Trägerverein oder »ein Nutzungsabkommen mit Einzelverträgen jede Gruppenstruktur bereits im Ansatz unmöglich machen würde« (Plenum 1997c: 3). Die Berliner Wagendörfer organisieren sich seit 1995 im wöchentlich tagenden Plenum der innerstädtischen Berliner Wagenburgen, die überregionale Koordination erfolgt seit 1990 im Quartalsrhythmus über die Wagentage. Wagentage finden in wechselnden Städten statt und dienen der Vernetzung der 120 dokumentierten Plätze in der BRD. Während der Wagentage wird die Zeitschrift Vogelfrai publiziert, die über Räumungsdrohungen, Verlagerungen, Umzüge u.ä. berichtet. Die Vogelfrai ist ausdrücklich nicht als Informationsblatt gedacht, sondern als internes Medium der BewohnerInnen. Diese Form der Gemeinschaftsbildung wird durch die Dauerhaftigkeit der einzelnen Standorte gewährleistet.

Die schwankende Bevölkerung der Wagendörfer in Berlin umfaßt einige hundert Personen in Abhängigkeit vom gesellschaftlichen und meteorologischen Klima. Die 1994 geschätzte Zahl von Ein- bis einigen Tausend BewohnerInnen in Berlin ist sicherlich zu hoch (Knorr-Siedow/Willmer 1994: 7). Das Plenum der innerstädtischen Wagenburgen kalkuliert im März 1997 ca. 600 BewohnerInnen an den damals 13 Standorten (Plenum, 1997c: 1), die ausnahmslos räumungsgefährdet sind. Bis auf zwei Standorte gelten alle anderen im März 1997 als illegale Siedlungen. Bei den legalisierten Standorten handelt es sich Ersatzflächen für geräumte Wagenburgen, die Anfang der 90er Jahre an der östlichen Peripherie ausgewiesen wurden.

Zusammenfassend sind Wagendörfer in ihrer sozialen Struktur, ihren Ent-

13 Auch diese Plätze genießen keine dauerhafte Vertragssicherheit. Gegen Auflagen und Pacht wird ein temporärer Aufenthalt gewährt, keine dauerhafte Nutzung. 
scheidungsfindungsprozessen, ihrer Binnensolidarität und ihrer Kritik an den dominanten Lebensformen mit Hauskollektiven oder großen Wohngemeinschaften vergleichbar. Mit dem allerdings fundamentalen Unterschied in der baulichen Form: »Allein die Organisation des Alltages ist beträchtlich zeitaufwendiger, als es das Leben in einer Geschoßwohnung erfordert« (Plenum 1997c: 3).

\section{Vom autoritären Sozialstaat zur strafenden Armenfürsorge: Zwei Räumungen}

»Ich bin nicht bereit, in dieser Stadt rechtsfreie Räume zu dulden« (Schönbohm, Senat für Inneres 1996a: 3)

Entstanden aus der Kritik an der Warenförmigkeit städtischen Lebens im Umfeld der Westberliner HausbesetzerInnenbewegung, siedeln einzelne Wagenplätze bereits seit Mitte der 80er Jahre auf dem zum Osten gehörenden aber nur vom Westen zugänglichen Mauervorland. Dieses Mauerland war einer der sensibelsten Räume im geteilten Berlin und durch komplexe völkerrechtliche Vereinbarungen reguliert. Jeder Vorfall, der die Integrität dieses Grenzgebietes in Frage stellte, hatte das Potential, internationale Verwicklungen zu provozieren. Geographisch konzentrierten sich die Siedlungen bis Mitte der 90er Jahre in und um den Bezirk Kreuzberg, der eine Infrastruktur von Arbeitsmöglichkeiten, sozialen Diensten und Solidarität bereithielt.

Mit dem Mauerfall und der Verschärfung der chronischen Krise des (West-) Berliner Wohnungsmarktes, realisierten die Wagenburgen die Chance, die diese Räume an der internen Peripherie zwischen Mauerfall und Wiedervereinigung boten. Seit der Vereinigung ist deren Verwertung zwischen unterschiedlichen Akteuren (Bund, Land, Alteigentümer, Bahn, Militär) heftig umstritten. 1990 besetzt eine Gruppe einen Teil des Mauerstreifens und wird für einen Tag von den polizeilichen Organen der DDR gegen die Westberliner KollegInnen geschützt. Danach greift die Vereinbarung der Polizeipräsidenten beider Stadthälften, daß die Westberliner Polizei »Extremisten und Störer « über den Mauerstreifen hinweg verfolgen darf (Telegraph, 4/90: 4). Die abrupte Verwandlung dieser einst ökonomisch peripheren Räume in erstklassiges Bauerwartungsland nährt Verwertungsgelüste unter Investoren und Politikern. Die Bodenrichtwerte der Grundstücke steigen 1994 in kerngebietstypischen Lagen wie der East Side (s.u.) auf 5.000-11.000 DM pro Quadratmeter (Gutachterausschuß 1995: Blatt 17). Die sozialdemokratische Variante der organisierten Verlagerung der Standorte an die städtischen Randzonen mit (befristeten) Nutzungsverträgen, Platzordnungen, Entgelten und Versorgungsinfrastruktur weicht einer Innenpolitik der Null-Toleranz. Bereits 1990 hatte der Senat beschlossen, 
keine weiteren Wagenplätze in der Innenstadt zu dulden und für die bestehenden Standorte Ersatzflächen auszuweisen. Im Frühjahr 1991 sichert die damalige Sozialsenatorin Stahmer (SPD) den BewohnerInnen des Wagendorfes am Potsdamer Platz ihre Unterstützung zu und der Kultursenator Roloff-Momin (parteilos) wünscht alles Gute (taz 15.4.91). Insgesamt aber wird ein harter Kurs eingeschlagen. An der Wagenburg am Engelbecken zwischen den Bezirken Kreuzberg und Mitte wird der neue Umgang mit sozial desintegrierten und vernachlässigten Wagenburgen vorexerziert. Die Wagenburg am Engelbecken bestand seit 1987 auf dem Mauervorland und galt als sozial schwächste der Berliner Wagendörfer. Nur 50\% der BewohnerInnen $^{14}$ erhalten Sozialhilfe, der Rest ist auf Betteln angewiesen. Auf dem Gelände existieren keine ausreichenden sanitären Anlagen. Unterstützung erfahren die BewohnerInnen nur von kirchlichen Kreisen, einzelnen Wohlfahrtsverbänden und lokalen Initiativen. Auf einer Pressekonferenz im Mai 1993 (»Mit Außenseitern leben«) werben die BewohnerInnen um Toleranz. Die kirchlichen Veranstalter weisen darauf hin, daß Dealer Platzverbot haben und der Schrott zu weiten Teilen vom Mülltourismus der NachbarInnen stammt. Schließlich haben Kühlschränke und Fernseher nur begrenzten Wert auf Plätzen, die nicht an das Stromnetz angeschlossen sind. Die Pressekonferenz endet desaströs, da ein Photograph der Welt gewaltsam daran gehindert wird, die Unterkünfte abzulichten (taz, 11.5.93).

Die Zusicherung des Bezirksamtes Mitte, die Wagenburg nicht zu räumen bevor der vorgesehene Wohnungsbau realisiert werde, wird gebrochen. Das Bezirksamt spricht am 7.10.1993 ein sofortiges Nutzungsverbot aus und läßt den BewohnerInnen eine Frist von 3 Stunden $^{15}$, um zu verschwinden. Die Anlässe für die Räumung sind bemerkenswert: Neben bau-, eigentumsund ordnungsrechtlichen Begründungen wird den BewohnerInnen vorgeworfen, daß die Anforderungen an gesunde Wohn- und Arbeitsverhältnisse nicht gewährleistet sind. Die Gefahr für die öffentliche Sicherheit und Ordnung wird damit begründet, daß ein »Verstoß gegen ungeschriebene gesellschaftliche Verhaltensregeln und Wertvorstellungen vor[liegt], die nach herrschender Anschauung als unerläßliche Voraussetzung für ein gedeihliches staatsbürgerliches Zusammenleben anzusehen sind« (Bezirksamt Mitte, 1993: 5). Weiter widerspricht die bauliche Form Wagenburg den »modernen Vorstellungen von einem geordneten Zusammenleben in einer Großstadt, zumal in der Innenstadt, insbesondere auch dem ästhetischen

14 Die EinwohnerInnenzahlen variieren in der Presse um den Faktor Zehn, es werden zu unterschiedlichen Zeitpunkten zwischen 40 und 400 BewohnerInnen gezählt. Der letzte Wert ist maßlos übertrieben.

15 Ausschlaggebend für dieses minimale Ultimatum war die Sorge, autonome Gruppen könnten den Platz besetzen und so die Räumung vereiteln (Bezirksamt Mitte 1993: 6f). 
Empfinden der übrigen Bevölkerung « (ebd.). ${ }^{16}$ Ungeschriebene Gesetze, moderne Vorstellungen und das ästhetische Empfinden der Bevölkerung sind Bedingungen, die über das Aufenthaltsrecht Armer, zumal in der Innenstadt, entscheiden. Die Begründung des Bezirksamtes unterscheidet sich von späteren Räumungen darin, daß die Voraussetzung für die Räumung die Bereitstellung eines angemessenen Ersatzgeländes und die Verhinderung von Obdachlosigkeit war. In späteren Verwaltungsdokumenten (s.u.) wird stets davon ausgegangen, daß die BewohnerInnen nicht obdachlos werden, sondern bereits wohnungslos sind und eine Räumung nichts an ihrem sozialen Status ändere.

Nach einer Pressekampagne, einem Seuchenalarm ${ }^{17}$ und der pauschalen Kriminalisierung der BewohnerInnen durch führende CDU-Politiker ${ }^{18}$ wird das Gelände am Engelbecken im Herbst durch 900 PolizistInnen geräumt. Ein Teil der BewohnerInnen zieht auf das Ersatzgelände nach Pankow, lehnt aber jede Fremdverwaltung ab: »Wir sind keine Treber, keine Obdachlosen, sondern Kulturträger für alternative Wohn- und Lebensformen und bestehen auf Selbstverwaltung und Eigenverantwortung « (taz, 13.10.93). Der Bezirk Pankow wehrt sich anfänglich vehement gegen die Ansiedlung, anonyme AnwohnerInnen hetzen auf Flugblättern gegen die WagenbewohnerInnen und im angrenzenden Ortsteil Karow gründet sich eine Initiative $»$ Für ein sicheres und sauberes Karow « (taz, 6.9.93). Drei Jahre später ist das Verhältnis zwischen Wagenplatz und Nachbarschaft jedoch entspannt (scheinschlag, 18/96: 3). Dezentral organisiert und in Eigenregie geführt, beherbergt der Platz ca. 100 BewohnerInnen. Auf dem Gelände am Engelbecken wird für zwei Millionen DM ein temporärer Sportplatz eingerichtet, der nach nur drei Jahren dem Wohnungsbau weichen muß (Morgenpost, 18.10.97).

Runde Tische, die von den Wagendörfern zur Lösung des Konfliktes seit August 1995 einberufen werden, ignoriert die Verwaltung. Daraufhin setzen die Wagenplätze die Gespräche im November aus.

Bei den Wahlen zum Abgeordnetenhaus im Herbst 1995 büßt die große Koalition über 400.000 Stimmen ein, bleibt aber an der Macht. Die Wahl

16 Die gestörte Ästhetik wird u.a. einerseits durch den Mülltourismus der NachbarInnen mitverursacht und ist andererseits der Tatsache geschuldet, daß die Wagen weder Stauraum noch Keller für Brennmaterial u.ä. bieten. Die Nutzung des Platzes zur Aufbewahrung der Bau- und Brennmaterialien stört die uninformierten Betrachter, die vermutlich nicht ohne Keller oder (Hänge-)Böden wohnen.

17 Der damalige Gesundheitsstadtrat Engelmann (CDU) dramatisiert: »Das Engelbecken ist Berlins schlimmste Müllhalde. Wenn hier nicht sofort geräumt wird, hat bald halb Kreuzberg die Gelbsucht« (Bild, 8.8.91).

18 Der Parlamentarische Geschäftsführer der CDU und Initiator des Anti-Graffiti-Vereins nofitti beschreibt die Wagenburg als »Schrottplatz« (Neue Zeit, 6.8.92), »Chaotendorf« und »innerstädtischen Schandfleck« (taz, 13.7.93). Die BewohnerInnen bilden »einen Querschnitt durchs Strafgesetzbuch« (Morgenpost, 4.8.92). 
markiert eine Zäsur. Die Olympiabewerbung war gescheitert, nur zögerlich siedeln sich Investoren an, der lokale Arbeitsmarkt kollabiert und immer mehr einkommensstarke Haushalte verlassen die Stadt. Der Senat forciert die Standortpolitik und erhöht den Druck gegenüber besetzten Häusern, Wagensiedlungen, Obdachlosen und einer Vielzahl von Verhaltensweisen und kultureller Praxen, die gegen mittelständische Standards verstoßen. Darunter fällt das Grillverbot in Parkanlagen und eine Vielzahl von Maßnahmen zur Verbesserung des Stadtbildes, ${ }^{19}$ unter anderem die Räumung von elf besetzten Häusern und Wagenburgen bis Mitte 1997 (taz, 13.6.97). Anfang 1998 gibt es in Berlin noch ein besetztes Haus.

In diesem Kontext wird eine einzelne Wagenburg am Ufer der Spree (EastSide-Wagenburg) primäres Objekt polizeilicher Intervention. Die Siedlung lag hinter dem international bekannten kilometerlangen Mauerstreifen, der von KünstlerInnen aus 21 Ländern gestaltet worden ist (East-Side-Gallery). Die soziale Desintegration an diesem Standort mit ca. 150 - 200 BewohnerInnen, war in erster Linie Resultat vorangegangener Räumungen ${ }^{20}$ und der Verweigerung sozialer Dienste gegenüber den EinwohnerInnen geschuldet. Obwohl das Gelände seit 1990 genutzt wird, stellt der Senat für Gesundheit und Soziales erst im Herbst 1994 wenige Toilettencontainer (10) auf. Im gleichen Jahr beginnen Treberhilfe und Berliner Tafel ${ }^{21}$ den Platz anzufahren, um die BewohnerInnen mit Nahrung und Hilfe zu versorgen. Es gibt weder Wasser noch Strom. Der Platz gilt als der sozial schwächste in Berlin. Im April 1996 treffen sich mehrere hundert WagenbewohnerInnen zu den überregionalen Wagentagen in Berlin, um sich gegen die Pathologisierung ihrer Lebensform zu wehren. Daraufhin verschärft sich die Kampagne gegen die East-Side. Im Vorfeld der Wagentage war es an der East-Side zu einem Mord an einem ortsfremden Jugendlichen auf der Suche nach Drogen gekommen, kurz darauf wurde ein weiterer Fremder verletzt. Wenige Tage später erklärt die Polizei das Gelände zum 'gefährlichen Ort' was den Entzug grundlegender Bürgerrechte für die BewohnerInnen bedeutet (vgl. Eick im vorliegenden Band). Unseres Wissens ist nie geklärt worden, ob der Mord und die Körperverletzung von BewohnerInnen begangen wurde. Konflikte um Drogen geraten auch an anderen Orten schnell außer Kontrolle. Es geht weder um die Bagatellisierung oder Beschönigung der Taten noch um die Leugnung gewaltförmiger sozialer Beziehungen auf der EastSide, sondern darum, daß diese mit der baulichen Form Wagenburg verknüpft werden. Die Realität der East-Side war zu diesem Zeitpunkt das Re-

19 Vgl. beispielsweise den Bericht zur Rettung des Stadtbildes (Senatsverwaltung für Jugend Schule und Sport 1997).

20 Aus der Kreuzberger Wilhelmstraße im Frühjahr 1990 und vom Engelbecken im Herbst 1993.

21 Eine private Mahlzeitennothilfe, die die Armen der Stadt kostenlos mit unverkaufter Nahrung versorgt. 
sultat eines Prozesses, der von Vernachlässigung, Ausgrenzung und Stigmatisierung der BewohnerInnen gekennzeichnet war. Weder die soziale Struktur noch die bauliche Form transformieren einen Raum zum Ghetto, sondern die Diskriminierung der BewohnerInnen. Die anderen Wagenplätze waren sich des dynamischen Slumcharakters der East-Side bewußt und prognostizierten, daß die dringend gebotene Lösung der Probleme durch eine Räumung nur verlagert werden würde (Berg 1997).

Die Presse kann mit den folgenden Razzien kaum Schritt halten, erweist sich aber als Instrument bei der Stigmatisierung des Platzes als outlaw ghetto. Die öffentliche, auch kritische Wahrnehmung, wurde durch die Medien, nicht die Erfahrung, geformt. Die mediale Repräsentation der Wagenburg als feindliches Kollektiv, das die städtische Gesellschaft durch Kriminalität, Drogen und Seuchen bedroht, war allgegenwärtig.

Politisch isoliert und fast ohne jede Unterstützung, verlassen die mobilen BewohnerInnen ab April den Platz. Mitte Juli wird auf dem Gelände die Leiche eines Bewohners entdeckt. Wie sich später klärt, war er an einer Lungenentzündung verstorben, doch der Verdacht auf Tuberkulose reicht als Räumungsanlaß zur Abwehr einer Gefahr für die öffentliche Sicherheit und Ordnung. ${ }^{22}$ Die verbliebenen 112 BewohnerInnen werden geräumt, 54 von ihnen haben keine deutsche Staatsbürgerschaft und sind nach der Räumung unerreichbar für Sozialarbeiter verschwunden. Die Presse schreibt von »zerzausten, zerlumpten, schmutzstarrenden Gestalten « auf der »bestbewachten Müllhalde der westlichen Hemisphäre« (Welt, 18.7.96), die BZ titelt mit »Drogen, Dreck und Tbc« (18.7.96) und der Tagesspiegel bringt die Einheit von Seuchen, Unrat und Drogen auf den Nenner: »Tuberkolose, Wracks und ein Cannabisfeld « (18.7.96).

Damit ist das Gelände frei für ein Projekt des Chicagoer Stararchitekten Helmut Jahn, der an dieser Stelle ein Büro- und Geschäftshaus mit dem Titel Spreefoyer für den Investor Opus plant. Das Gelände der Wagenburg lag auf einem geplanten Uferweg und einem Spreepark, der in der Planung des Investors von einem öffentlichem Raum zu einem »kleinem Freigehege für die Mittagspausen der Büroarbeiter « einschrumpft (taz, 6.7.95). Ein Jahr nach der Räumung wird das Gelände als Baustofflager genutzt und kostenintensiv bewacht. Von Investitionen keine Spur.

22 Die Seuchengefahr war allerdings tatsächlich nur der Anlaß. Maßgeblich war der privatrechtliche Anspruch des Bundesvermögensamtes, mit dem die Senatsinnenverwaltung einen Räumungstermin verabredet hatte (Tagesspiegel 18.7.96). Die Oberfinanzdirektion, die das Bundesvermögen in Berlin verwaltet, war allerdings nicht glücklich mit diesem Arrangement und fühlte sich zum Räumungsantrag erpreßt (Scheinschlag 18/96) 


\title{
Die gescheiterte Regulation metropolitaner Armut an der Peripherie
}

\author{
»Je weiter wir aus dem Inneren heraustreten, desto politischer wird die At- \\ mosphäre. Es kommen die Docks, die Binnenhäfen, die Speicher, die Quar- \\ tiere der Armut, die zerstreuten Asyle des Elends: das Weichbild. Weichbil- \\ der sind der Ausnahmezustand der Stadt, das Terrain auf dem ununterbro- \\ chen die große Entscheidungsschlacht zwischen Stadt und Land tobt « (Wal- \\ ter Benjamin, 1928: 63)
}

Diese große Entscheidungsschlacht zwischen Zentrum und Peripherie gegen die Asyle des Elends wurde in Berlin $1996 \mathrm{f}$ geschlagen. Während der Konflikt um die East-Side kaum Solidarität von der städtischen Linken erfuhr, entfachte der Beschluß des Senates, den BewohnerInnen ein temporäres Ersatzgelände ${ }^{23}$ an der westlichen Peripherie zuzuweisen, eine ausgewachsene NIMBY-Rebellion. Der gewählte Standort in Staaken-West unterscheidet sich nur scheinbar von den anderen Ersatzstandorten, die ausnahmslos an der östlichen Peripherie liegen. Administrativ gehört der Ortsteil Staaken-West (4000 EinwohnerInnen) zum Bezirk Spandau. Zwar wurde das Dorf 1920 nach Großberlin eingemeindet, doch nahmen die britischen Alliierten mit der Sowjetunion 1945 einen Gebietsaustausch zugunsten eines Militärflughafen vor. Trotzdem überließ die Sowjetunion zwischenzeitlich Westberlin die Verwaltung des Gebietes. Erst 1951 besann sich die DDR ihrer Hoheitsrechte und besetzte den Ort durch die Volkspolizei. In unmittelbarer geographischer Nähe zu Berlin, doch abgelegen von den Arbeits- und Wohnungsmärkten der Hauptstadt der DDR, wird der Ort mit dem Vereinigungsvertrag wieder der Stadt zugeschlagen. Das zeitigt kuriose juristische Konsequenzen wie die temporäre Fristenregelung bei Schwangerschaftsabbrüchen (taz, 21.12.90). Laut Zeitungsberichten fühlen sich die BewohnerInnen nicht annektiert, sondern sind stolz auf ihren neuen Status als Großstädter (ebd.), Großstadt ja, auch Hauptstadt, aber nicht Metropole: Die Entscheidung des Senates zur Ausweisung dieses Ersatzstandortes traf keineswegs auf eine fragmentierte Machtstruktur an der geographischen Peripherie, sondern auf die geschlossene Front von BewohnerInnen, deren Meinung über die East-Side von der vorangegangenen Kampagne der Medien geformt und gefestigt worden war. »Staaken zittert vor den Rollheimern« titelt der Berliner Kurier (18.7.96). Der entmachtete Bezirk Spandau ${ }^{24}$ und die AnwohnerInnen wehren sich

23 Die Ausweisung eines Ausweichstandortes hatte sich seit Monaten hingezogen und war in sämtlichen Bezirken an Protesten der AnwohnerInnen und Bezirksämter (Pankow, Weißensee, Hohenschönhausen) gescheitert. Im Frühsommer beginnt die Sozialverwaltung exterritoriale, berlineigene Flächen in Brandenburg (sog. Stadtgüter) zu prüfen, was dort auf Argwohn stößt (Berliner Zeitung, 28.6.96).

24 Der Bezirksbürgermeister Birkholz (CDU), wurde wenige Tage vor der Räumung von einem Staatssekretär parteiintern über das geplante Ausweichgelände informiert und mußte sich auf »kurze, heftige Unfreundlichkeiten beschränken« (Bezirksamt Spandau 1996: 
gegen diese Arroganz der Macht, die aus ihrer Perspektive metropolitane Armut in ihren Vorgärten entsorgt (BVV Spandau 1996, Bürgerinitiative 1996). Ca. 100 BewohnerInnen zwingen fünf anreisende Wagen zur Umkehr, indem sie sie mit Hämmern und Fäusten begrüßen (Tagesspiegel 25.7.96). Obwohl die meisten BewohnerInnen der East-Side sich der Einlieferung in das 'Internierungslager' widersetzen, müssen sie innerhalb von 14 Tagen einen legalen Standort nachweisen, um ihre konfiszierten Besitztümer und Wagen von der Polizei zurückzuerhalten. Während die geräumten BewohnerInnen in anderen Wagensiedlungen unterkommen oder verstreut über die Innenstadt temporäre Siedlungen formen, beweisen die EinwohnerInnen von Staken-West die Zentralität der Peripherie. Sie bilden eine Bürgerinitiative und formen eine mächtige Koalition aus $\mathrm{CDU},{ }^{25}$ Bund der Steuerzahler, verschiedenen Senatsverwaltungen, anrainenden Privateigentümern, die sich weigern, den Wagenburgen das Wegerecht zu gewähren, Abwasserverbänden und anliegenden Umlandgemeinden bis hin zur direkten Unterstützung durch den Regierenden Bürgermeister Diepgen (CDU). Die Sozialverwaltung zeigt sich unbeeindruckt: »Niemand kann uns den Zugang zu unserem Land verwehren. Notfalls richten wir eine Luftbrücke ein« (Berliner Kurier 30.7.96).

Noch im Oktober überreicht die Bürgerinitiative Diepgen mehr als 13.000 Unterschriften (taz, 1.11.96). Im Frühjahr 1997 folgt das Abgeordnetenhaus einer Empfehlung des Petitionsauschusses und läßt alle Pläne für ein Ersatzgelände in Staaken-West oder anderswo fallen, um die jungen, hausbesitzenden Familien mit kleinen Kindern vor genau den Zumutungen zu schützen, wegen derer sie unter Inkaufnahme erheblicher finanzieller Belastungen aus der Innenstadt geflüchtet sind: »Nach Ansiedlung einer Wagenburg wären die Kinder nicht mehr sicher « (AH 1996g: 11). Die WagenbewohnerInnen werden der Fürsorge bezirklicher Einrichtungen der Wohnungslosenhilfe überantwortet. Doch nur wenige sind verzweifelt genug, dieses Angebot wahrzunehmen.

Die letzte Zuflucht für ca. 30-50 verbliebene BewohnerInnen aus Deutschland, Großbritannien, Italien, Frankreich und Polen auf einer ungenutzten Straße zwischen den Bezirken Mitte und Kreuzberg wird mittels der StraBenverkehrsordnung in der Osterwoche 1997 geräumt: Der betreffende Straßenabschnitt wurde Montagabend zur Parkverbotszone erklärt und

10). Der Senat reagiert auf die anschließende Kritik nicht. Hauptkritikpunkt der Bezirksverordnetenversammlung ist die Entmachtung des Bezirkes und die Entziehung des Grundstückes aus dem bezirklichen Finanzvermögen durch die Hauptverwaltung.

25 So beschließt der CDU-Landesverband (1996): »Weder im innerstädtischen Bereich noch an der Landesgrenze kann es Bestandsgarantien für Obdachlose oder auch andere Bürger in Wagendörfern auf Kosten der Steuerzahler geben.« Der Fraktionsvorsitzende Landowsky will generell keine Einrichtungen der Wohnungslosenhilfe in der Nähe von Wohnsiedlungen dulden (Morgenpost 19.8.96). 
Mittwochmorgen geräumt.

Es ist zweifelhaft, ob die Verhinderung der Ansiedlung dem Erfolg der Bürgerinitiative in Staaken zuzuschreiben ist. Die überwiegende Mehrheit der WagenbewohnerInnen wollte und konnte nicht umsiedeln. Die vital wichtige Nähe zu sozialen Dienste, Arbeitsmärkten und solidarischen Strukturen der Innenstadt verunmöglichte die Verlagerung. Die Aussicht auf einen temporären Standort (drei Monate) inmitten einer feindlichen Umwelt mit Zugangskontrollen, Zwangsattesten, überwachter Einhaltung der Platzordnung und vor allem einen Kostenbeitrag von 550 DM monatlich pro Wagen taten ein Übriges (AH 1996b: 1). Es darf allerdings bezweifelt werden, daß der Ersatzstandort je eine ernstgemeinte Option der Hauptverwaltung war. Innensenator Schönbohm erklärte unmittelbar nach der Räumung, die Verlagerung nach Staaken sei eine »Fiktion« (Welt 19.7.96). Bausenator Klemann (CDU) erklärt, er habe mit der Wagenburg »nichts am Hut« und halte die Verlagerung »für eine übertriebene Fürsorge Berlins«. Er blockiert offen das Genehmigungsverfahren (Berliner Zeitung 27.11.96). Stadtentwicklungssenator Strieder (SPD) stellt sich wenig später ebenfalls gegen das Projekt. Sozialsenatorin Hübner (CDU) verlautbart im September 1996, das Gelände in Staken sei eine Drehscheibe, von der aus die WagenbewohnerInnen auf ein zukünftiges Gelände der Berliner Stadtgüter in Brandenburg vermittelt werden sollten (Berliner Zeitung 16.9.96). Damit befinden sich die wichtigsten betroffenen Verwaltungen in Opposition zum Senatsbeschluß der Verlagerung. Im April 1997 beschließt der Senat unter Hinweis auf die prekäre finanzielle Lage der Stadt, das Gelände in Staaken fallenzulassen, nachdem bereits 55.000 DM investiert worden sind. ${ }^{26}$ Bei zukünftigen Räumungen sollen keine Ersatzflächen mehr ausgewiesen, sondern die bezirkliche Wohnungslosenhilfe beansprucht werden (taz 30.4.97).

Die Rolle der Bürgerinitiative in diesem Konflikt bleibt widersprüchlich. Gegen die mediale Gleichsetzung der BI mit einzelnen Knüppelschwingern, hat sie sich stets verwahrt. Nach Auskunft einer Gründerin, wird die Initiative von Müttern und Hausfrauen getragen, die sich sehr schnell das professionelle Protestrepertoire angeeignet haben. Zudem stehen die Datschen- und SiedlungsbewohnerInnen in Staken selbst unter erheblichen Restitutionsdruck (scheinschlag 18/96: 3). Die gemeinsame Interessenlage mit den WagenbewohnerInnen resultierte nicht in einer taktischen Allianz gegen den Senat und für die Duldung innerstädtischer Plätze. Statt dessen verteidigt die BI ihre territorialen Interessen in der Allianz mit dem dominanten Ausgrenzungsdis-

26 Davon 40.000 DM für die Errichtung eines Zaunes und 10.000 DM für Vermessungsarbeiten (AH 1996a). Insgesamt wurden Kosten von 2 Millionen Mark kalkuliert, die allerdings soweit wie möglich auf die Entgelte der BewohnerInnen umgelegt werden sollten. 
kurs. Diese Ehe mit den hegemonialen Kräften gebärt das erwünschte Kind: »Wir hatten viel Streß. Aber es ist wie bei einer Schwangerschaft. Wenn man es überstanden hat, ist vieles vergessen «, so die Iniatorin der Bewegung (Berliner Zeitung 2.5.97). Die Initiative erweist sich als wirkliche NIMBYBewegung. Ihr militanter Partikularismus wendet sich gegen jegliche bauliche Verdichtung und mobilisiert nun mit einer weiteren BI gegen eine dänische Investorengruppe, die auf dem Gelände einen riesigen Tivoli-ThemePark für 700 Millionen DM errichten will (Berliner Zeitung 8.3.97).

\section{Die Herstellung der Hauptstadtfähigkeit}

»Die Räumung der East-Side-Gallery ist nach der Räumung der besetzten Häuser ... ein weiterer Schritt zur Behebung von schwerwiegenden Mißständen, welche das Ansehen Berlins und den Stolz der Berliner auf ihre Stadt empfindlich beeinträchtigen « (Schönbohm, in: Der Senat für Inneres 1996a: 3)

Die Räumung der East-Side war ein Signal an alle Wagenplätze und besetzten Häuser. Der Senat kündigte mit der Räumung an, sämtliche bestehenden Wagendörfer, also auch die legalisierten Standorte, bis Ende des Jahres 1997 zu räumen. Während die East-Side ein stets gefährdeter defensiver Raum der städtischen Armutsbevölkerung war, stammen die politisch aktiven und sozial stabilen Wagenplätze in und um Kreuzberg aus der Tradition der HausbesetzerInnenbewegung. Diese Artefakte einer vergangenen partizipatorischen Planungskultur stellen ein Hindernis bei der Verwertung innerstädtischer Räume dar und stören das Leitbild der Dienstleistungsmetropole. In den Augen des parlamentarischen Geschäftsführers der CDU Hapel sind sie nichts weiter als bewohnte Müllkippen (taz 13.7.95). Der Innensenator Schönbohm beschreibt die Räumung als Teil einer Strategie, die unruhige Metropole zu normalisieren: ${ }^{27} \gg$ In diesem Sinne ist die Räumung der East-Side ein weiterer Schritt auf dem Weg zur Normalität und zur Stärkung der Hauptstadtfähigkeit Berlins « (Senatsverwaltung für Inneres 1996a: 3). Mit der Entscheidung, keine weiteren Ersatzstandorte auszuweisen, bricht der Senat entschlossen mit der Berliner Linie und reorganisiert die Geometrie der Macht, indem er die Verantwortlichkeit für Wagenplätze an die Bezirke abgibt. Die Berliner Linie ist (oder besser: war) das lokale Arrangement zwischen Kommunalpolitik und HausbesetzerIn-

27 Die Notwendigkeit solcher Normalisierungen wird nicht von allen Eliten geteilt. So sieht der Siemens Vorstand Martinsen keine Beeinträchtigung des Standortes Berlin durch Wagenplätze, HausbesetzerInnen oder linksradikale Szenen: »Sicherlich kann man das Thema kontrovers diskutieren. Aber eine Stadt zeigt Vitalität, wenn sie verschiedene Strömungen toleriert und mit ihnen lebt. In Hausbesetzungen sehe ich keine Beeinträchtigung des Standortes Berlin« (taz 19.8.93). Er betont damit weniger die Toleranz der industriellen Investoren als den relativen Bedeutungsverlust urbaner sozialer Bewegungen in Berlin. 
nenbewegung. Es war nicht unbedingt ein Dokument des Erfolges der HausbesetzerInnenbewegung, doch es bot verläßliche Regelungen und Verfahren für den Fall einer Besetzung oder Räumung. Die Berliner Linie ist nicht offiziell außer Kraft gesetzt, sondern weiter Teil der Koalitionsvereinbarung zwischen SPD und CDU (Koalitionsvereinbarungen 1995: 61), aber die Praxis des Senates, ein Ersatzgelände für geräumte Wagensiedlungen auszuweisen, ist mit dem Senatsbeschluß vom 29.4.97 obsolet. Damit werden Wagenplätze aus der Tradition urbaner sozialer Bewegungen herausgelöst und administrativ als hilfsbedürftige Wohnungslose redefiniert. Wir haben uns in der Darstellung des Konfliktes auf die ordnungspolitische Praxis konzentriert. Dagegen ließe sich einwenden, daß offiziell nicht der Senator für Inneres, sondern die Senatorin für Gesundheit und Soziales für Wagenburgen zuständig ist. Doch spätestens 1996 entwickelt sich das Problemfeld Wagenburgen zu einer ressortübergreifenden Aufgabe: Der Senat für Finanzen stellt das Grundstück in Staaken zur Verfügung, der Senator für Bau, Wohnungswesen und Verkehr prüft die Genehmigungsfähigkeit, der Senat für Stadtentwicklung, Umweltschutz und Technologie erteilt befristete Ausnahmegenehmigungen für das Wasserschutzgebiet in Staaken und der Senator für Inneres räumt. Die Wahrnehmung der immer wieder betonten Angebote der Sozialverwaltung hätte bedeutet, daß die BewohnerInnen sich der Armenfürsorge anvertrauen, die weite Teile der Wohnungslosenhilfe kennzeichnet. Bedingung der Hilfegewährung ist die Anerkennung der herrschenden gesellschaftlichen Normen und eines rigiden Zeitregimes. In diesem Sinne ist das Ausweichgelände in Staaken als eingezäuntes, kontrolliertes, temporäres und überwachtes Einschließungsterrain nicht das Gegenteil von Sozialpolitik, sondern das extreme Ende eines Kontinuums, das Verhaltensanpassung zur Bedingung von Hilfegewährung macht. Auf die Frage eines Wagenplatzes, was die BewohnerInnen denn nun genau zu Obdachlosen mache, antwortet die Sozialsenatorin u. a.: Das Fehlen von Verträgen. Die Köpenikiade des fin de millenium: Obdachlos ist, wer keinen (Miet-) Vertrag hat und einen Vertrag erhält nicht, wer als wohnungslos definiert ist.

\section{Innerstädtisches Territorium im Bedeutungswandel}

»Innerstädtisch besagt, innerhalb der Berliner Stadtgrenze« (Hübner 1997)

Diese eigenwillige Definition der Senatorin für Soziales ist auf die Wagenplätze gemünzt (AH 1997), beschreibt aber gut die Standortpolitik des Senates gegenüber allen städtischen Flächen. Freiflächen, Brachen, öffentliche Räume usw. werden samt und sonders für Investitionen mobilisiert. Insbesondere das große und heterogene Areal innerhalb des inneren S- 
Bahnringes (der sog. Hundekopf), das konventionell »Innenstadt « genannt wird, ist aus der Perspektive des Senates primärer Raum, der durch primäre Nutzungen verwertet werden muß. Die ausgedehnten industriellen Brachlandschaften, die der Deindustrialisierungsproze $\beta$ der Treuhand hinterlie $\beta$, mit den eingestreuten Archipelen der leerstehenden Büro- und Einzelhandelsflächen an den Knotenpunkten der S-Bahn, lassen Zweifel aufkommen an der behaupteten Hochwertigkeit aller Flächen. Standorte für Wagenplätze gäbe es genug, aber die Weigerung des Senates, Flächen für solche konkurrierenden Nutzungen auszuweisen, ist nicht allein der Arroganz der Macht geschuldet, sondern verweist auf die unterschätzte Möglichkeit marginalisierter Gruppen und unerwünschter Nutzungen, im Urbanisierungsprozeß die Umwertung der Bedeutung von Räumen vorzunehmen und ihrer eigenen Stigmatisierung entgegenzuwirken.

Wie oben beschrieben ist Marginalisierung keine statische Kategorie, sondern eine dynamische Strategie. Die Marginalisierung ausgewählter Gruppen, Nutzungen und Räume mag ein Projekt der lokalen Wachstumskoalition zur Herstellung eines urbanen Konsenses sein, das spezifische Produkt ist jedoch Resultat sozialer Auseinandersetzungen und politischer Kämpfe. Das Verständnis des Urbanisierungsprozesses ist integraler Bestandteil des Verständnisses polit-ökonomischer und sozialer Prozesse und Probleme. Daher ist der Konflikt um die Berliner Wagenplätze nicht auf die BewohnerInnen begrenzt, sondern artikuliert die Krise der Stadt: Die ausgedehnten innerstädtischen Industriebrachen zeugen von der ökonomischen Transformation; der ordnungspolitische Zugriff auf die Wagenburgen ist ein Versuch, der sozialen Polarisierung politisch Herr zu werden und nur ein Beispiel für die eskalierenden Ausgrenzungsprozesse aus öffentlichen Räumen, dem Wohnungs- und Arbeitsmarkt.

Die Wagenplätze sind nicht hilflose Opfer der Berliner Stadtentwicklungspolitik, sondern zeichnen sich durch den stets gefährdeten, doch gegenwärtig erfolgreichen Versuch aus, ihre Stigmatisierung als gesetzlose Desperados abzuwehren. Dank einer Reihe von Aktionen wie Demonstrationen, Öffentlichkeitsarbeit, Tage der offenen Tür, Diskussionsveranstaltungen, Presseerklärungen, Runde Tische, Lobbying von Mitgliedern des Abgeordnetenhauses bis hin zu Gesprächen mit dem Innensenator und der Wahrnehmung ihrer Interessen im formalen Planungsprozeß, konnten sie das öffentliche Labeling als unwürdige Obdachlose teilweise abwehren und statt dessen ihr Selbstverständnis als kollektive Lebensform betonen. Unter anderem durch den Verweis auf die Disziplin, die es erfordert, bei begrenztem Zugang zu Strom und Wasser unter Berliner Witterungsbedingungen in Wagen zu leben, erlangten die Wagenplätze vorläufige und prekäre Sympathien. Seit Ende 1996 sind die Presseberichte weniger feindlich. Selbst ein so mächtiger Akteur wie die Oberfinanzdirektion (OFD), die die 
Grundstücke des Mauerstreifens verwaltet, erklärt, keine Räumungsklage einzureichen (Oberfinanzdirektion 1996: 1): »Uns ist eine funktionierende Wagenburg lieber als ein leeres Grundstück, das wir für viel Geld bewachen lassen müßten $\ll(B Z 14.8 .96){ }^{28}$

\section{Lokale Reaktionen: Bezirksämter, AnwohnerInnen und InvestorInnen}



Dieser Wahrnehmung der Wagenplätze als feindliche und unkontrollierbare Kollektive, die den städtischen Gesamtkörper bedrohen, mögen sich die verantwortlichen Bezirksämter nicht länger anschließen. 1997 deuten sich erste Lösungen an. Die Mehrzahl der innerstädtischen Bezirke steht dabei im offenen Widerspruch zur Senatspolitik. Die Bürgermeisterin des Großsiedlungsbezirkes Hohenschönhausen Grygier (für PDS) hätte zum Schrecken der lokalen CDU und besorgter WählerInnen gerne eine Wagensiedlung. Allerdings geriet auch hier der Kontakt zwischen WagenbewohnerInnen und KleingärtnerInnen ${ }^{29}$ zum Fiasko (MieterMagazin 12/97: 15). Die WagenbewohnerInnen des Vereins Engelbecken $e$. V. werben vergeblich um Verständnis: »Wir wollen nicht anders leben als Schrebergärtner. Nur, daß wir in unseren Wagen auch übernachten wollen« (Berliner Zeitung, 21.8.97). Das Büro der Bürgermeisterin teilte mit, daß wegen der besondere Sensibilität der Annäherung beider Gruppen die Öffentlichkeit vorläufig ausgeschlossen bleibe. Der Kreuzberger Bürgermeister Schulz (Bündnis'90/Die Grünen) erneuert gegenüber den beiden Wagenplätzen vor Ort die Duldung. Der Bezirk hält gegen die CDU an der Kreuzberger Linie fest, die Bestandsschutz für bestehende Siedlungen garantiert (Berliner Zeitung 16.4.97). Der Bezirk Friedrichshain verlagert in Absprache mit dem Investor $O M G$ (Hotel), einem Staatssekretär (Kurth, CDU) ${ }^{30}$ und den Wa-

28 Noch zwei Wochen zuvor hatte die OFD die Räumung aller Wagenplätze auf den Liegenschaften der Behörde angekündigt (Welt 10.8.96), wenige Tage später weicht sie jedoch von dem Konfrontationskurs ab. Die OFD überläßt die Räumungsklagen den zukünftigen privaten Eigentümern, an die die Liegenschaften nach dem Gesetz über den Verkauf von Mauer- und Grenzgrundstücken an die früheren Eigentümer verkauft werden sollen. Über den Einfluß einer Demonstration der Wagenplätze vor dem Sitz der OFD auf diese Entscheidung kann nur spekuliert werden.

29 Die WagenbewohnerInnen verweisen regelmäßig ironisch auf ihre Nähe zu den SchrebergärtnerInnen. Beide Nutzungen sind Sondernutzungsflächen, die im formalen Planungsprozedere nicht vorgesehen sind. Allerdings sind die 100.000 Kleingärtner Berlins ausgesprochen normenkonform und verfügen mit Angehörigen und Sympathisanten über 500.000 Stimmen. Noch kein Politiker hat ernsthaft erwogen, diese wertvollen innerstädtischen Flächen in großem Maßstab für andere Nutzungen zur Verfügung zu stellen: »Wer Kleingärtner quält, wird abgewählt« (taz 18.10.95).

30 Investor und Finanzverwaltung teilen sich die Kosten für die Säuberung der Ersatzfläche 
genbewohnerInnen den Standort innerhalb des Bezirkes und handelt sich damit die Klage einer Investorengruppe ein, die auf dem Nachbargrundstück 60 Eigentumswohnungen errichten will. Die NachbarInnen laufen gegen die Umsiedlung Sturm und der Verfassungsschutz unterrichtet das Bezirksamt, daß die Republikaner sich diese Stimmung zu Nutze machen wollen (Morgenpost 11.9.97). Die Klage einer Nachbarin, die die Verwertung ihres Eigentums durch den Wagenplatz gefährdet sieht, wird im August als unbegründet abgewiesen. Die klagenden Investoren haben mehr Erfolg. Das Gericht weist das Bezirksamt an, ein Nutzungsverbot auszusprechen und dieses sofort zu vollziehen (Berliner Zeitung 26.11.97). Damit sind die bereits ausgehandelten Pachtverträge mit den WagenbewohnerInnen hinfällig. Im Bezirk Treptow setzt die CDU handstreichartig die Räumung der »integrationsunwilligen Obdachlosen« durch, die wiederum von dem Betreiber eines benachbarten Vergnügungsparks medienwirksam einen Stellplatz angeboten bekommen. Die WagenbewohnerInnen sollen auf dem Gelände Jugendarbeit ihres Vereines (KulturBanausen e.V.) anbieten, wie schon im ganzen Bezirk (Berliner Zeitung 1.10.97). Sie sammeln über 4.000 Unterschriften in der Nachbarschaft für den Erhalt des Platzes, verhandeln aber auch mit dem Bezirk Lichtenberg über einen neuen Standort.

Sowohl die Befürchtungen der Investoren und der AnwohnerInnen als auch die Hoffnungen des Vergnügungsparkbetreibers verweisen darauf, daß Wagendörfer und andere Marginalisierte in der Lage sind, die Bedeutung urbaner Räume aufzuwerten, abzuwerten oder gar zu entwerten. Diese mögliche Transformation der Bedeutung städtischer Räume durch unerwünschte NutzerInnen ist die Ratio hinter den Vertreibungsprozessen in den deutschen Innenstädten. Dies widerspricht der Annahme, daß die Marginalität von Räumen die Marginalität der NutzerInnen reproduziere. Die Kontrolle des Zuganges zu ihren Plätzen und die Definition der sozialen Bedeutung dieser Plätze sind Beispiel und Bedingung strategischer Raumkontrolle (s. u.), die Wagenplätze von den meisten anderen Marginalisierten unterscheidet - zumindest im Moment.

\section{Strategisches Handeln und taktisches Verhalten - die Verhandlung von Aufenthaltsrecht in der Innenstadt}

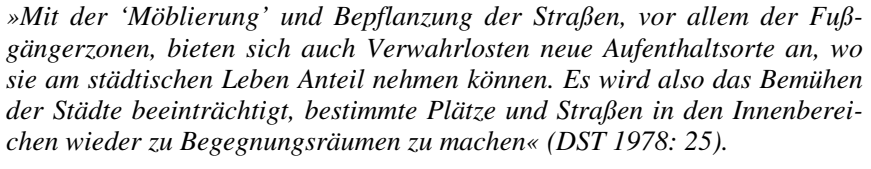

»Mit der 'Möblierung' und Bepflanzung der Straßen, vor allem der Fußgängerzonen, bieten sich auch Verwahrlosten neue Aufenthaltsorte an, wo sie am städtischen Leben Anteil nehmen können. Es wird also das Bemühen der Städte beeinträchtigt, bestimmte Plätze und Straßen in den Innenbereichen wieder zu Begegnungsräumen zu machen «(DST 1978: 25).

Der Städtetag beklagte früh die Verwahrlosung der Innenstädte durch die

in Höhe von DM $70.000(\operatorname{taz} 25.7 .97)$ 
Präsenz Obdachloser, die alle Urbanitätsbemühungen zunichte zu machen drohten. Die relativen und prekären Erfolge der Wagenplätze bilden keine einmalige Ausnahme. In Berlin und anderswo finden sich weitere Beispiele der Aushandlung territorialer Kompromisse (vgl. Schmid 1996), in denen z.B. Obdachlose und ihre BetreuerInnen gegen die Stigmatisierung der Verwahrlosung Aufenthaltsrecht erstreiten.

Wagendörfer genießen das Privileg strategischer Raumkontrolle gegenüber taktischer Raumaneignung (Ruddick 1996: 58ff). Strategisches Handeln ruht auf der Kalkulation von Machtbeziehungen und der planvollen Umsetzung von normativen Orientierungen im Konflikt. Taktisches Verhalten findet im Konflikt selbst statt, es ist eher raffiniert als kalkuliert. Eine Taktik nimmt Gelegenheiten wahr, wie sie sich bieten und hängt von der Anwesenheit und Identifizierung solcher Gelegenheiten ab. Taktiken arbeiten mit dem cleveren Gebrauch der Zeit, ohne räumliche Basis. Strategien operieren von kontrollierten Räumen aus. Der Erwerb und die Sicherung solch selbstbestimmter Territorien macht Strategien möglich, ihr Verlust macht Taktiken nötig. Militaristisch formuliert wird Taktik in der Schlacht und Strategie im Krieg angewandt.

Solch geschickter und kalkulierter Gebrauch der Zeit, zeigt sich in den Raumaneignungspraxen Wohnungsloser. Ein Beispiel ist die Taktik, der gesellschaftlichen Stereotypisierung als Obdachloser zu entgehen, indem die Betreffenden sich als solche unsichtbar machen, um Zugang zu öffentlichen Räumen zu finden. Damit werden nur scheinbar bestehende Strukturen reproduziert, denn diese Taktik stellt eine Verhandlung dar, deren Ziel es ist, den Raum für Zwecke zu nutzen, die nicht vorgesehen sind - das Überleben in der Innenstadt. ${ }^{31}$ Aus dieser Perspektive erscheint die Unstetigkeit oder Unfaßbarkeit Obdachloser nicht als psychologischer Mangel und Ursache der Wohnungslosigkeit, sondern als Überlebenstaktik Unterprivilegierter. Nur dieser prekäre, transitorische Gebrauch von Räumen sichert den Obdachlosen relative Permanenz an zentralen Orten. Wohnungs-

31 Da an dieser Stelle der Platz fehlt, das Argument für Wohnungslose weiterzuverfolgen, führen wir hier nur ein Beispiel an: Der Bahnhof Zoo ist nach der Privatisierung der Bundesbahn zu einem stark kontrollierten Ort geworden. Das Bemühen der Bahn AG, die soziale Bedeutung des Raumes Bahnhof von traditionellen Konnotationen zu befreien, scheint erfolgreich. In den komplett kommodifizierten Bahnhofshallen ist vorgeblich kein Platz mehr für Wohnungslose und andere, deren Anwesenheit mit monatlich Tausenden Bahnhofsverweisen allein am Zoo geahndet wird (vgl. Eick im vorliegenden Band). Dieser Schein trügt. Auf der dem Bahnhof abgewandten Seite des Zoos hat sich ein komplexer Servicecluster etabliert. Diese sozialen und medizinischen Dienste sind nicht stationär, sondern mobil. Die Fahrzeuge bieten zu regelmäßigen Zeiten am Zoo ihre Dienste an. Die Entscheidungen über die Standorte werden in einem der Senatsinnenverwaltung zugeordneten Gremium namens CITY AG gefällt, das bedarfsabhängig die relevanten AkteurInnen (z.B. Bahn AG, Einzelhandelsvereinigung, Anbieter sozialer Dienste usw.) versammelt und Standorte zuweist. 
lose sind nicht a priori auf den taktischen Gebrauch von Räumen zurückgeworfen. In den Straßenzeitungsprojekten finden sich Beispiele, in denen die soziale Position Obdachloser redefiniert, Konzessionen von Autoritäten erhandelt und Zugänge zu Ressourcen eröffnet werden. Diese erlauben es, dem Diktat täglicher Bedürfnisse zu entkommen und wieder langfristige Ziele zu verfolgen. Die im Umfeld der Straßenzeitungen entstehenden Wohn- und Arbeitsprojekte bieten die Basis für die Etablierung strategischer Raumkontrolle.

Noch aber sind es in erster Linie die Wagenplätze, die den Zugang, die Nutzung und die soziale Bedeutung ihrer Standorte definieren und kontrollieren. Diese Fähigkeit ist keine inhärente Qualität der baulichen Form Wagendorf, sondern muß ständig durch die BewohnerInnen und UnterstützerInnen reproduziert werden. Sie ist nicht nur durch direkte Repression gefährdet, sondern ebenso durch die Verlagerung der Standorte an die städtische und soziale Peripherie. Die Selbstwahrnehmung der Wagendörfer ist das Resultat ihrer kollektiven Anstrengung, ihre Räume zu definieren und zu kontrollieren und ihre Plätze an das breitere Spektrum alternativer Lebensentwürfe anzuschließen. Wenn diese homologe Beziehung zwischen Räumen, Identitäten und der Subkultur nicht mehr gewährleistet ist, kann aus der Lebensstilentscheidung Wagenplatz schnell eine bloße Adaptionsstrategie gegenüber Obdachlosigkeit an den geographischen und sozialen Rändern der Stadt werden. Die Wagendörfer sind sich der komplexen Beziehungen zwischen ihren Identitäten, ihrer Subkultur und den Räumen, in denen sie leben, bewußt. Bedingung ihrer Verhandlungsbereitschaft mit dem Senat ist nicht das Beharren auf den gegenwärtigen Standorten, ${ }^{32}$ sondern darauf, daß alle Ersatzangebote in der Innenstadt innerhalb des inneren S-Bahnringes liegen müssen. Die geographischen Imaginationen der Sozialsenatorin (s.o.) lassen keinen Zweifel daran, daß der Senat nicht gewillt ist, diese Bedingung zu akzeptieren. Der feste Räumungswille wird weitere Wohnungslose produzieren und den Konkurrenzdruck in peripheren Räumen und um überforderte Dienste verschärfen. Die Kritik an der Warenförmigkeit städtischen Lebens wird so zum mangelnden Vermögen, in dieser Stadt zu leben, umdefiniert.

Die Repräsentationspolitiken der Wagenplätze sind ein Versuch, dieses gut entwickelte Vermögen zu reproduzieren und vertraglich zu fixieren. Obwohl kein nachhaltiger Durchbruch zu verzeichnen ist, sind die zögerlichen

32 Das wäre die beste Lösung, da an den etablierten Standorten aufgrund langjähriger Kontakte kaum Konflikte mit den NachbarInnen auftreten und die BewohnerInnen in Schulen, Vereine und Nachbarschaften integriert sind. »Warum sollte mensch diese gewachsenen Strukturen zerstören und an anderer Stelle Menschen ungefragt (!) eine Wagenburg vor die Tür setzen, welche Sie auf keinen Fall wollen?« (Plenum der Berliner innerstädtischen Wagenplätze 1997c: 4). 
Signale der Bezirke ein Beleg, daß sich die entschlossene Intervention der Wagenplätze in die lokale Politik trotz aller Niederlagen und Rückschläge gelohnt hat. Immerhin stehen gegen den erklärten Willen des mächtigen Innensenators noch 11 Wagenplätze in der Stadt (Stand: Januar 1998). Als das größte Hindernis bei dem Ringen um Anerkennung als berechtigte BewohnerInnen der Innenstadt formulieren die Wagenplätze den ständigen Rechtfertigungsdruck: »After all, the biggest conflict is the permanent need to justify ourselves in the face of pressure, to open ourselves to and provide services for the general public « (Berg 1997: 5). Nur einige größere Hauskollektive bieten noch ein vergleichbares Niveau an nicht-warenförmigen Angeboten (Zirkus, Varieté, Feste, Konzerte usw.) und niemand käme auf die Idee, von Zehlendorfer VilleneignerInnen den Zutritt zu ihren Grünflächen zu verlangen, wie er von den Wagenplätzen im allgemeinen gewährt wird. $\mathrm{Ob}$ es sich tatsächlich um (sicherlich nicht gleichberechtigte) Aushandlungsprozesse handelt oder um die bloße Zuweisung immer nur geduldeter Standorte und Standzeiten, die jederzeit widerrufen werden können, muß hier offen bleiben. Politische und wissenschaftliche Analysen jedoch, die a priori den universellen Geltungsanspruch metropolitaner Standortlogik anerkennen, sind diesem Urbanisierungsprojekt schon erlegen und arbeiten an dessen Ausdehnung mit. Diese Ausdehnung bedeutet eskalierende Ausgrenzung: aus dem Arbeitsmarkt, aus dem Wohnungsmarkt, aus dem öffentlichen Raum, aus der städtischen Gesellschaft und damit aus dem Geltungsbereich sozialer Bürgerrechte.

Wie der Konflikt um die Berliner Wagenburgen und Wagenplätze innerhalb der gespannten Beziehung zwischen Zentrum und Peripherie zeigt, ist Urbanisierung ein widersprüchlicher Prozeß ungleicher und unbestimmter Entwicklung. »Obwohl hegemonial, kann die Logik der Kapitalakkumulation niemals jede Nuance der Urbanisierung kontrollieren« (Harvey 1997: 6). In dieser Ungleichheit und Widersprüchlichkeit lokaler Politiken und hegemonialer Projekte liegt die Möglichkeit und Notwendigkeit politischer Intervention jenseits der formalen Beteiligungsgremien.

\title{
Das revanchistische Berlin
}

\author{
"Kriminelle, Asoziale und sonstige unnormale Menschen, die ihre Daseins- \\ berechtigung höchstens auf isolierten Stadtgütern in der Mark Brandenburg \\ fristen dürften « (Landowsky)
}

So qualifiziert der Fraktionsvorsitzende der CDU die WagenbewohnerInnen ab und verbannt sie rhetorisch in Reservate (zitiert nach: Kropp 1997: 3.5.1.). Diese Wiederkehr gefährlicher Klassen (Kriminelle, Asoziale, sonstige unnormale Menschen) an gefährdeten Orten wird neuerdings auch 
hierzulande als die Formation einer urban underclass kontrovers diskutiert (Bremer/Gestring 1997, Häußermann 1997b, Kronauer 1996, Siebel 1997). Die neue Qualität sozialer Spaltung, die damit beschrieben wird, variiert lokal. Die Restratifizierung von Räumen und Gruppen in Berlin ist kein Prozeß, der allein marktförmig über das Bodenregime und über den $\mathrm{Ar}$ beitsmarkt vermittelt wird. Dieser Prozeß wird von einer lokalen Politik getragen, die Neil Smith revanchistische Stadtpolitik nennt (1996: 189-232, 1996b, c). Damit beschreibt er eine Politik der Restauration gegenüber den Errungenschaften urbaner sozialer Bewegungen und des lokalen Sozialstaates als Bedingung und Konsequenz ökonomischer und politischer Expansion der städtischen Zentren. Kennzeichnend für diesen Revanchismus ist die Transformation liberaler Sympathie mit den Marginalisierten zur Verteidigung von Privilegien und Subventionen in Zeiten scharfer Budgetkürzungen. Wenn es weniger Güter und Leistungen zu teilen gibt, soll auch mit immer Wenigeren geteilt werden, die ihre Berechtigung als legitimierte Anspruchsberechtigte zu beweisen haben. Der Kreis der legitimen EmpfängerInnen wird dynamisch enger gezogen und der Leistungsbezug an Normenkonformität geknüpft. Wer den Kriterien nicht genügen kann oder will fällt dem strafenden Staat (Mragequpdätzk9gel)termhiaindiesem Kontext trotz ihrer wiederholten Zahlungsbereitschaft als mietstreikende Parasiten, die wertvollen Raum widerrechtlich besetzen. Sie werden als urbane Anomalie des späten, hochsubventionierten Westberlin begriffen, deren reine Anwesenheit einen Imageschaden für die nationale Bedeutung Berlins darstellt. »Berlin ist nicht Hauptstadt der Wehrdienstverweigerer, nicht Hauptstadt der Wagenburgen, nicht Hauptstadt der Hausbesetzer, sondern Berlin ist die Hauptstadt der Deutschen « stellt der ehemalige General und Ernst Jünger-Liebhaber Schönbohm klar (AH 1996h: 995). In dieser Stadt sind alle Berliner normenkonforme Deutsche, deren standortrelevante Sekundärtugenden Schaden nehmen könnten an der Alternative eines selbstbestimmten urbanen Lebens in Würde mit wenig Geld, wie es die Wagenplätze vorleben.

\section{Literatur}

AH (Abgeordnetenhaus von Berlin) 1996a: Kleine Anfrage 13/1070 der Abgeordneten Heike Ließfeld (SPD) über bisher entstandene und noch zu erwartenden Kosten für die Herrichtung des Ersatzstandortes in West-Staaken für die Eastside Wagenburg und Antwort der Senatorin Hübner (30.12.96), Berlin.

- 1996b: Kleine Anfrage Nr. 893 der Abgeordneten Ruth Mecklenburg (SPD) über Wagenburg in West-Staaken und Antwort der Senatorin Hübner (12.9.96), Berlin.

- 1996c: Inhaltsprotokoll der 10. Sitzung des Ausschuß für Soziales (9.9.96), Berlin.

- 1996d: Kleine Anfrage 13/1339 der Abgeordneten Ruth Mecklenburg (SPD) über die geplante Wagenburg in West-Staaken und Antwort der Senatorin Hübner (30.12.96), Berlin.

- 1996e: Inhaltsprotokoll der 12. Sitzung des Ausschusses für Inneres, Sicherheit und Ord- 
nung (14.10.96), Berlin.

- 1996f: Inhalts- sowie Beschluß-Protokoll der 14. Sitzung des Ausschusses für Bau- und Wohnungswesen U. a. zum Abbau der Fehlsubventionierung im Berliner Wohnungswesen sowie zur Fehlbelegungsabgabe (30.10.96), Berlin.

-1996g: Votum des Petitionsausschusses des Abgeordnetenhauses von Berlin zum Standort Staaken für eine Wagenburg, in: Drucksache 13/1234, Berlin.

- 1996h: Große Anfrage der Fraktion Bündnis 90/Die GRÜNEN über Häuserräumungen, Auflösungen von Wagenburgen - Wohin treibt der Säuberungswahn den Senat? (20.8.96), in: Drucksache 13/664, Berlin.

- 1997: Kleine Anfrage 13/2445 der Abgeordneten Marion Seelig (PDS) über Senatsbeschluß zu Wagenburgen und Antwort der Senatorin Hübner (9.7.97), Berlin.

AK - Analyse und Kritik, ehemals Arbeiterkampf.

Architektenkammer Berlin (Hg.) 1997: Planwerk Innenstadt Berlin: Eine Provokation, Berlin.

Baroner, Wolfgang 1996: Eigenheimbau in Berlin stärker fördern, Neuformulierung eines politischen Schwerpunktes. (unveröffentlichtes Ms.).

Becker, Ruth 1997: Im Labyrinth der Wohnungspolitik. Anmerkungen zu Mieten, Belastungen, Förderungsmodellen und Subventionsformen. Ist der soziale Wohnungsbau obsolet geworden?, Ms. Beitrag für einen Ausstellungskatalog des BDA in Hamburg.

Benjamin, Walther 1929: Denkbilder. Frankfurt/Main: Suhrkamp, 1994.

Berg, Renate 1997: Islands in a Coded Public Space, in: INURA (Hg.): Possible Urban Worlds - The Reader of the $7^{\text {th }}$ INURA Conference 1997 (im Erscheinen).

Berliner Mietergemeinschaft 1997: Emanzipation von dem Mietern. In: MieterEcho. Zeitung der Berliner Mietergemeinschaft, Nr. 263, Juli/August 1997.

Bezirksamt Mitte 1993: An die Bewohner der »Wagenburg « auf dem ehemaligen Grenzstreifen Legiendamm/Ecke Waldemarstraße/Ecke Dresdener Straße am Engelbecken. In: KnorrSiedow, Thomas; Walther Willmer 1994: Sozialverträglicher Umgang mit unkonventionellen, mobilen Wohnformen am Beispiel des Wohnens in Wohnwagendörfern und Wagenburgen. Berlin: IRS

Bodenschatz, Harald 1987: Platz frei für das neue Berlin. Geschichte der Stadterneuerung in der größten Mietskasernenstadt der Welt seit 1871. Berlin.

Bremer, Peter; Norbert Gestring 1997: Urban Underclass - neue Formen der Ausgrenzung in deutschen Städten?, in: PROKLA 106, S. 55-76.

Bundesministerium für Raumordnung, Bauwesen und Städtebau 1996: Siedlungsentwicklung und Siedlungspolitik. Nationalbericht Deutschland zur Konferenz HABITAT II. Bonn.

Bürgerinitiative West-Staaken 1996 (Hg.): Informationsbroschüre gegen die Ansiedlung einer Wagenburg.

BVV (Bezirksverordnetenversammlung) Spandau 1996: Protokoll der Sitzung vom 16.8.96 u.a. das Ausweichgelände für die Wagenburg Staaken betreffend.

CDU Landesverband 1996: Beschlüsse in Sachen Wagenburgen, Pressemitteilung (19.8.96).

Dear, Michael; Mahs, Jürgen von 1994: Genesis I Evaluation. Final Report. Los Angeles: University of Southern California.

- 1997: Housing for the Homeless, By the Homeless, And of the Homeless, in: Ellin, Nan (Hg.), Architecture of Fear. Princeton: Architectural Press.

Deutscher Städtetag (DST) 1978: Stadtstreicher. Kommunale Erfahrungen, Probleme, Antworten. In: Reihe B. DST-Beiträge zum Kommunalrecht, Heft 3. Köln: Deutscher Städtetag.

Foyer, siehe Senatsverwaltung für Bauen, Wohnen und Verkehr.

Garreau, Joel 1991: Edge City. Life on the new Frontier. New York: Doubleday.

Grell, Britta; Sambale, Jens; Veith, Dominik 1997: Inner!City!Action! - Crowd Control, Interdictory Space and the Fight for Sociospatial Justice, in: INURA (Hg.): Possible Urban Worlds - The Reader of the $7^{\text {th }}$ INURA Conference 1997 (im Erscheinen).

Gutachterausschuß für Grundstückswerte in Berlin, Senatsverwaltung für Bau- und Wohnungswesen (Hg.) 1995: Bodenrichtwerte 21.12.1994.

Hain, Simone 1996: 'Nun wächst zusammen, was zusammengehört!'? Erster Kommentar zum Planwerk. Referat zur 60. Sitzung des Stadtforums (29.11.96).

Harvey, David 1997: The New Urbanism and the Communitarian Trap, in: Harvard Design Review No. 1 (Winter/Spring), S.5-6. 
Häußermann, Hartmut 1997a: Was ist eine Innenstadt?, in: Architektenkammer Berlin (Hg.) 1997: Planwerk Innenstadt Berlin: Eine Provokation. Berlin, S. 64- 67.

- 1997b: Armut in den Großstädten - eine neue städtische Unterklasse?, in: Leviathan 1. S. 12-27.

Innen!Stadt!Aktion! 1997: Gegen Privatisierung, Sicherheitswahn und Ausgrenzung. Zeitungsbeilage der Innen!Stadt!Aktion! erschienen in: Berner Tagwacht, scheinschlag, taz und WoZ.

Keil, Roger 1993: Weltstadt - Stadt der Welt. Internationalisierung und lokale Politik in Los Angeles. Münster: Westfälisches Dampfboot.

Knorr-Siedow, Thomas; Walther Willmer 1994: Sozialverträglicher Umgang mit unkonventionellen, mobilen Wohnformen am Beispiel des Wohnens in Wohnwagendörfern und Wagenburgen. Berlin: IRS.

Koalitionsvereinbarung zwischen der Christlichen Demokratischen Union Deutschlands (CDU) Landesverband Berlin und der Sozialdemokratischen Partei Deutschlands (SPD) Landesverband Berlin (1995), Berlin.

Kronauer, Martin 1996: 'Soziale Ausgrenzung' und 'Underclass': Über neue Formen der gesellschaftlichen Spaltung, in: SOFI-Mitteilungen Nr. 24. S. 53-69.

Kropp, Herbert 1997: Wagenleben - Das Leben wagen. Empirische Studie über das Leben und Wohnen in fahrbaren Behausungen, Diplomarbeit an der Universität Oldenburg.

MieterEcho, siehe Berliner Mietergemeinschaft.

Oberfinanzdirektion 1996: Weiteres Vorgehen der OFD Berlin gegenüber den Wagenburglern auf dem ehemaligen Mauerstreife, in: Pressemitteilung, 15/96 (26.8.96), Berlin.

Plenum der Innerstädtischen Berliner Wagenplätze 1997a: Berlin braucht Freiräume für alternatives Wohnen - Wagenplätze in Berlin. Flugblatt (Februar 1997).

- 1997b: Stadt-Ab-Wicklung oder Alternatives Wohnen. Flugblatt (Mai 1997).

- 1997c: Freiräume für alternatives Wohnen - Wagenplätze für Berlin. Vortagsmanuskript für das 9. Stadtforum von Unten (3.3.97).

Ronneberger, Klaus 1997a: Bronx in Deutschland. Vortragsmanuskript für die Konferenz: Peripherie oder neue Stadt? Zur Amerikanisierung der Stadtentwicklung, Bauhaus Dessau, (1.-3.5 97)

- 1997b: Peripherie - die Zukunft des Städtischen? In: Nachrichtenblatt zur Stadt- und Regionalsoziologie 1, S. 8 - 14.

Ruddick, Susan 1996b: Young and Homeless in Hollywood. Mapping the Social Imaginary. London: Routledge.

Sambale, Jens 1995: 'Racism comes in Birkenstocks' - Städtischer Umweltschutz und ethnische Risikodiskriminierung, in: Sträter, Frank 1995: Stadt der Zukunft-Zukunft der Stadt. Los Angeles - Berlin. Stuttgart: context. S. 66-77.

Sambale, Jens; Veith, Dominik 1997: Marginalisierung als urbaner Prozeß, in: Forschungsjournal Neue Soziale Bewegungen 2. S. 100-103.

Schmals, Klaus M., Jahn, Walther 1997: Simultanpolitik durch Stadtforen? - Das Beispiel Berlin, in: Heinelt, Hubert; Mayer, Margit (Hg.) 1997: Modernisierung der Kommunalpolitik. Neue Wege der Ressourcenmobilisierung. Opladen: Leske \& Budrich S. $192-215$.

Schmid, Christian 1996: Urbane Region und Territorialverhältnis - Zur Regulation des Urbanisierungsprozesses, in: Bruch, Michael; Krebs, Hans-Peter (Hg.) 1996: Unternehmen Globus. Facetten nachfordistischer Regulation. Münster: Westfälisches Dampfboot, S.224-254.

Schubert, Manuela 1997: Wagendörfer. Eine alternative mobile Wohnform, in: Bauwelt, Heft 38. S. $2136-2137$.

Schulz, Marlies 1991: Der Tauschwohnungsmarkt in der zentralistischen Planwirtschaft. Das Beispiel von Ostberlin, in: ISR-Forschungsberichte, Heft 3. Wien.

Senatsverwaltung für Bauen, Wohnen und Verkehr 1997: Berlin zu Hause. Eigentum in der Hauptstadt, in: Foyer, Magazin der Senatsverwaltung für Bauen, Wohnen und Verkehr, 3/97.

Senatsverwaltung für Inneres 1996a: 'Wagenburg an der East-Side-Gallery wird heute geräumt', Pressemitteilung Nr. 49 (17.07.1996).

- 1996b: Pressemitteilung Nr. 54 (24.7.96) zu Senatsbeschluß zu Wagenburgen.

Senatsverwaltung für Schule, Jugend und Sport, 1997: Bericht zur Rettung des Berliner Stadtbildes.

Senatsverwaltung für Stadtentwicklung, Umweltschutz und Technologie (Hg.) 1997a: Plan- 
werk Innenstadt. Ein erster Entwurf, in: Stadtentwicklung, Band 4. Berlin: Kulturbuchverlag.

- 1997b: Emanzipation der Mieter, in: Stadtforum. Journal der Senatsverwaltung für Stadtentwicklung, Umweltschutz und Technologie, No. 25.

Senatsverwaltung für Wirtschaft und Betriebe (Hg.) 1996: Berlin - Räume für die Wirtschaft.. Siebel, Walter 1997: Armut oder Ausgrenzung? Vorsichtiger Versuch einer begrifflicher Eingrenzung der sozialen Ausgrenzung, in: Leviathan 1/97. 67-75.

Smith, Neil 1996a: The New Urban Frontier. Gentrification and the Revanchist City. London: Routledge.

- 1996b: Social Justice and the New American Urbanism: The Revanchist City, in: Merrifield, Andy; Swyngedouw, Erik (Eds.), The Urbanization of Injustice. London: Lawrence \& Wishart, S. 117 - 137.

- 1996c: After Tompkins Square Park: Degentrification and the Revanchist City, in: King, Anthony (Hg.) 1996: Re-presenting the City. Ethnicity, Capital and Culture in the 21stCentury Metropolis. New York: New York University Press, S. 93-110.

Soja, Edward 1992: Inside Exopolis. Scenes from Orange County, in: Sorkin, Michael (Hg.) 1992: Variations on a Theme Park. The New American City and the Event of Public Space. New York City The Noonday Press. S. $94-122$.

Stadtforum, siehe Senatsverwaltung für Stadtentwicklung, Umweltschutz und Technologie.

Stephan, Eric 1997: EPIC ENTER. Crashing in Cars. In: San Francisco Bay Guardian, May 7th. S. 24-26.

tageszeitung/scheinschlag (Hg.) 1996: stadt.plan.mitte. Gemeinsame Beilage von scheinschlag und taz zur Diskussion über das Berliner 'Planwerk Innenstadt'. (November 1996).

Veith, Dominik; Sambale, Jens 1997: Mythos öffentlicher Raum - oder: Warum der Kampf um die Innenstadt wichtig ist. In: Arranca! 12 (Juni).

Wacquant, Loïc J. D. 1997: Vom wohltätigen zum strafenden Staat: Über den politischen Umgang mit dem Elend in Amerika. In: Leviathan 1/97. S. 50-66.

Wagenburg Schwarzer Kanal 1997: Die Wagenburg Schwarzer Kanal erfüllt wichtige soziale, kulturelle, ökologische, politische und städtebaulich wertvolle Funktionen im Bezirk Mitte. Flugblatt.

Wagendorf Lohmühle 1997: Neuigkeiten über Wagenburgen, Flugblatt.

Wagenplatz am Kinderbauernhof 1996: Selbstdarstellung. 11 Seiten.

Wolff, Katrin 1996: Umwandlung von Miet- in Eigentumswohnungen in Berlin. Ursachen, Umfang und Folgen, in: ISR Diskussionsbeitrag Nr. 45. ISR: Berlin.

\section{PROKLA 112 (September 1998): Europa (Teil I: Osteuropa)}

In zwei Heften wird sich PROKLA mit dem Thema Europa beschäftigen. Im Osten ist die Transformation der ehemals »realsozialistischen« Länder in kapitalistische Marktwirtschaften weit vorangeschritten, ohne daß sie damit schon zum Abbild Westeuropas geworden wären. Ein spezifisch »politischer Kapitalismus« hat sich herausgebildet. Auf der anderen Seite befinden sich auch die westeuropäischen Länder in tiefgreifenden Umbruchsprozessen sowohl ihrer wohlfahrtsstaatlichen Strukturen als auch mit der Einführung des Euro - ihrer politischen und wirtschaftlichen Beziehungen untereinander. Regionale Unterschiede werden zunehmen, während sich die Rolle der Nationalstaaten erheblich verändern wird. Damit verschieben sich auch die Bedingungen unter denen Gewerkschaften, soziale Bewegungen und politische Parteien ihre Auseinandersetzungen führen. 
